\title{
REVIEW
}

\section{A Review of Novel Combinations of Colistin and Lipopeptide or Glycopeptide Antibiotics for the Treatment of Multidrug-Resistant Acinetobacter baumannii}

\author{
Kimberly C. Claeys • Anna D. Fiorvento • Michael J. Rybak
}

To view enhanced content go to www.infectiousdiseases-open.com

Received: October 6, 2014 / Published online: December 5, 2014

(C) The Author(s) 2014. This article is published with open access at Springerlink.com

\section{ABSTRACT}

As antibiotic resistance continues to increase among Gram-negative organisms such as Acinetobacter baumannii there is a growing need for novel therapies to overcome these resistance mechanisms. Antibiotics active against multidrug-resistant $A$. baumannii (MDRAB) are few, and agents in development are primarily active against other multidrugresistant Gram-negative organisms. The combinations of colistin and antimicrobials such as glycopeptides and lipopeptides are

Electronic supplementary material The online version of this article (doi:10.1007/s40121-014-0051-9) contains supplementary material, which is available to authorized users.

K. C. Claeys - M. J. Rybak ( $\varangle)$

Anti-Infective Research Laboratory, Eugene Applebaum College of Pharmacy and Health Sciences, Wayne State University, Detroit, MI 48201, USA

e-mail:m.rybak@wayne.edu

K. C. Claeys · M. J. Rybak

Department of Pharmacy Practice, Eugene

Applebaum College of Pharmacy and Health

Sciences, Wayne State University, Detroit,

MI 48201, USA

A. D. Fiorvento

Detroit Receiving Hospital, Detroit Medical Center, 4201 St Antoine St, Detroit, MI 48201, USA unique potential treatment modalities against MDRAB. For both lipopeptides and glycopeptides in vitro data have demonstrated significant synergy, resulting in rapid bactericidal activity in time-kill curves. Several invertebrate in vivo models have also demonstrated increased survival compared to colistin alone. Currently, very little clinical data have focused on using these combinations for infections caused exclusively by multidrugresistant Gram-negatives. The combination of vancomycin and colistin has been studied with conflicting results regarding both improved outcomes and risk of nephrotoxicity. Although in vitro and in vivo models have proved promising, further investigation is required to provide clinical data necessary to support the use of these combinations. The objective of this review is to summarize literature currently available for the novel combination of lipopeptides or glycopeptides with colistin for the treatment of $A$. baumannii, in particular MDRAB.

Keywords: Acinetobacter baumannii; Combination therapy; Daptomycin; 


\section{INTRODUCTION}

Antibiotic resistance continues to be a global public health crisis [1]. The Centers for Disease Control and Prevention (CDC) state that resistant bacteria cause over two million infections in the United States annually, with a mortality rate of over 20,000 individuals per year $[2,3]$. Curtailing resistance through infection control measures is key to mitigating the threat and decreasing the spread of these organisms [4]. Once patients are infected, however, optimizing antimicrobial therapy becomes paramount. This is a challenge secondary to the declining availability of active agents and dwindling pipeline of novel antibiotics. As such, antibiotic-resistant bacterial infections can result in significant morbidity and mortality, often requiring the use of second- or third-line antibiotic agents that are more toxic and/or less effective. According to the CDC's Antibiotic Resistance Threat Assessment, multidrug-resistant Acinetobacter baumannii (MDRAB) pose a serious threat that may become urgent without public health monitoring and intervention [2]. Although there are currently antimicrobials in the later stages of development with promising activity against drug-resistant Gram-negatives, the activity against MDRAB is minimal $[5,6]$. The Infectious Diseases Society of America (IDSA), in their update entitled Bad Bugs, No Drugs: No ESKAPE, states that Acinetobacter baumannii (ACB) is "emblematic of the mismatch between unmet medical needs and current antimicrobial research..." [7]. This is echoed in the 2010 update of the IDSA's $10 \times 20$ Initiative that shows eight antimicrobials in phase II or III development with none demonstrating activity against MDRAB [8]. With the rise of multidrug, extensively drug, and pandrug-resistant
Acinetobacter spp. there is a critical need to discover safe and effective therapeutic strategies.

\section{The Challenge of Acinetobacter Infections}

Initially considered to have low pathogenicity, $\mathrm{ACB}$ is now one of the most important nosocomial pathogens, especially intensive care unit (ICU)-associated infections such as bacteremia, pneumonia, and wound infections. Often a colonizer of skin and sputum, ACB also has the ability to live on fomites, such as ventilator equipment, for prolonged periods of time [9]. Clinically, ACB infections have demonstrated increased lengths of mechanical ventilation, hospital/ICU stays, and mortality rates of $30-70 \%$ [9-12]. These organisms often harbor multiple resistance mechanisms through both selective pressures secondary to antibiotic exposure and transmission from other highly resistant organisms. A recent genomic study of Acinetobacter spp. found that these organisms has the ability to acquire over 40 resistance genes from other pathogenic Gram-negative bacteria [13]. Three main resistance mechanisms permit ACB to circumvent antimicrobial treatment: hydrolyzing enzymes, such as beta-lactamases; condensed porin channel proteins on its surface that decrease target entry sites; and alteration of cellular functions and antimicrobial targets [14]. Increasingly, multidrug- and extensively drugresistant ACB isolates have been reported, with the incidence of carbapenem resistance exceeding $75-90 \%$ in some Asian countries [15]. There are limited therapeutic options for carbapenem-resistant isolates and these infections are associated with a high morbidity and mortality. Merely repurposing existing FDA-approved agents to treat carbapenemresistant ACB has proved less than favorable 
[16]. Polymyxin antimicrobials have lost favor in treating infections secondary to toxicities associated with higher doses $[17,18]$. These agents are generally considered a last resort for the treatment of multidrug-resistant Gramnegative infections such as carbapenemresistant $\mathrm{ACB}$, or after other treatments have proven ineffective. Colistin, a cationic polypeptide, interacts with anionic lipopolysaccharide (LPS) layer of Gramnegative bacteria leading to osmotic lysis of the cell. Research has focused primarily on combination therapy versus monotherapy with colistin as the primary active agent. Promising in vitro results have been demonstrated with colistin in combinations with fosfomycin, tigecycline, and carbapenems. Clinical results, primarily associated with the endpoint of mortality, have been mixed [19-22]. This review will focus on several unconventional combinations with colistin that have demonstrated possible clinical utility.

\section{METHODS}

Literature associated with the combination of colistin and glycopeptides, lipopeptides, and lipoglycopeptides for the targeted treated of ACB was included in this review. All relevant English-language peer-reviewed publications were accessed through PUBMED using the following medical subject heading (MeSH) terms: vancomycin, daptomycin, telavancin, glycopeptide, lipopeptide, lipoglycopeptide, colistin, Acinetobacter baumannii, and combination therapy. Citations within these references were also assessed for inclusion. Publications in abstract form were included in the review if these abstracts were presented as part of professional meetings.
The analysis in this article is based on previously conducted studies and does not involve any new studies of human or animal subjects performed by any of the authors.

\section{DISCUSSION}

\section{Unconventional Approaches to Treatment}

\section{Studies with Glycopeptide Antibiotics}

Until recently little was known about combinations of colistin and antimicrobials with exclusive activity against Gram-positive bacteria. Novel combinations of colistin plus glycopeptide and lipopeptide antibiotics have recently been examined, yielding positive results [23, 24]. Vancomycin is a glycopeptide antibiotic that has become a mainstay in the treatment of methicillin-resistant Staphylococcus aureus (MRSA) over the past two decades that works by inhibiting peptidoglycan synthesis in the bacterial cell wall $[25,26]$. The proposed mechanism of synergy relates to the cellmembrane permeabilizing ability of colistin. Colistin causes an electrostatic interaction with LPS (lipid A), disrupting the outer membrane of the Gram-negative bacteria. This allows the otherwise large and hydrophobic vancomycin molecules to pass through the outer LPS layer and reach the site of action at the cell wall $[23,24]$.

Synergy between vancomycin and colistin in MDRAB was first described by Gordon et al. [23] with in vitro checkerboard assays, synergy Etests, and time-kill curves, against 39 MDRAB isolates; five epidemic strains and 34 clinical, and one reference isolate [American type culture collection (ATCC) 19606]. For the checkerboard assays, synergy was first defined by the lowest fractional inhibitory concentration index (FICI) of $\leq 0.5$. The FICI 
was calculated as [(minimum inhibitory concentration $[\mathrm{MIC}]$ colistin with vancomycin)/(MIC colistin alone $)+($ MIC vancomycin combined with colistin)/(MIC vancomycin alone)]. The second method was the two-well method wherein the absence of growth in wells containing $0.25 \times$ MIC of both drugs and $2 \times$ MIC of both drugs was deemed synergistic. These tests were completed on the five epidemic strains and ATCC 19606. Synergy by the Etest method was tested as the change in vancomycin MIC in colistin-supplemented versus unsupplemented agar plates. Time-kill curves against the five epidemic strains were conducted with colistin alone and with the combination of colistin and vancomycin at amounts that mimic steady-state concentrations at standard dosing-colistin $1 \mathrm{mg} / \mathrm{L}$ and vancomycin $20 \mathrm{mg} / \mathrm{L}$. Samples were removed as $1 \mathrm{~mL}$ aliquots at times $0,2,4$, 8,24 , and 48 . Synergy was found by both the checkerboard assay and the two-well method, demonstrating a decrease in vancomycin MIC in four of the six tested isolates. Etest synergy was performed on all isolates. Vancomycin MICs were $>256 \mathrm{mg} / \mathrm{L}$ on unsupplemented agar, while MICs in the presence of colistin ranged from 48 to $<0.016 \mathrm{mg} / \mathrm{L}$. In the time-kill curves of the five epidemic strains, colistin alone was initially bactericidal, however, regrowth occurred as early as $4 \mathrm{~h}$. The combination was bactericidal and was able to suppress regrowth during the entire $48 \mathrm{~h}$ of incubation for all except one isolate. This isolate was plated and demonstrated a sevenfold increase in colistin MIC. A similar in vitro study was conducted using teicoplanin, a glycopeptide antibiotic with a mechanism of action similar to vancomycin [27]. Again, significant in vitro synergy with colistin was demonstrated in checkerboard assays, synergy Etests, and time-kill curves. Several other in vitro studies of similar design have also demonstrated synergy between either vancomycin or teicoplanin and colistin (Table 1 [23, 24, 27-31]) [28-30]. Of note, the study by Vidaillac et al. demonstrates the enhanced activity of the combination in ACB compared to other Gram-negative isolates (Fig. 1 [29]). Additionally, significantly enhanced survival has been demonstrated with in vivo Galleria mellonella invertebrate models during combination therapy in the treatment of ACB infections [32]. The survival rate of larvae inoculated with MDRAB treated with colistin monotherapy was $48 \%$, compared to $>90 \%$ in the vancomycin combination and $67 \%$ in teicoplanin combination.

Although the combination of glycopeptide antibiotics and colistin has demonstrated promising in vitro and in vivo results there are concerns regarding its clinical application; in particular, the risk of combining two agents with known risk of nephrotoxicity. To date there have been few clinical studies directly evaluating the efficacy and safety of this combination. In a retrospective review of patients who received at least 5 days of combination therapy against MDRAB investigators noted no significant difference in ICU length of stay or 28-day mortality (48\% versus 50\%) [33]. This lack of clinical correlation may be secondary to the small sample size of 57 patients. There was, however, still a significant increase in the risk of developing renal failure across any acute kidney injury network (AKIN) stage $(p=0.04)$. The combination was then studied in a larger cohort of critically ill patients infected with Gram-negative bacteria, including, but not limited to, patients with MDRAB [34]. Of the 184 patients included, 99 patients were infected with MDRAB, 48 received the combination of glycopeptide and colistin. The remaining 


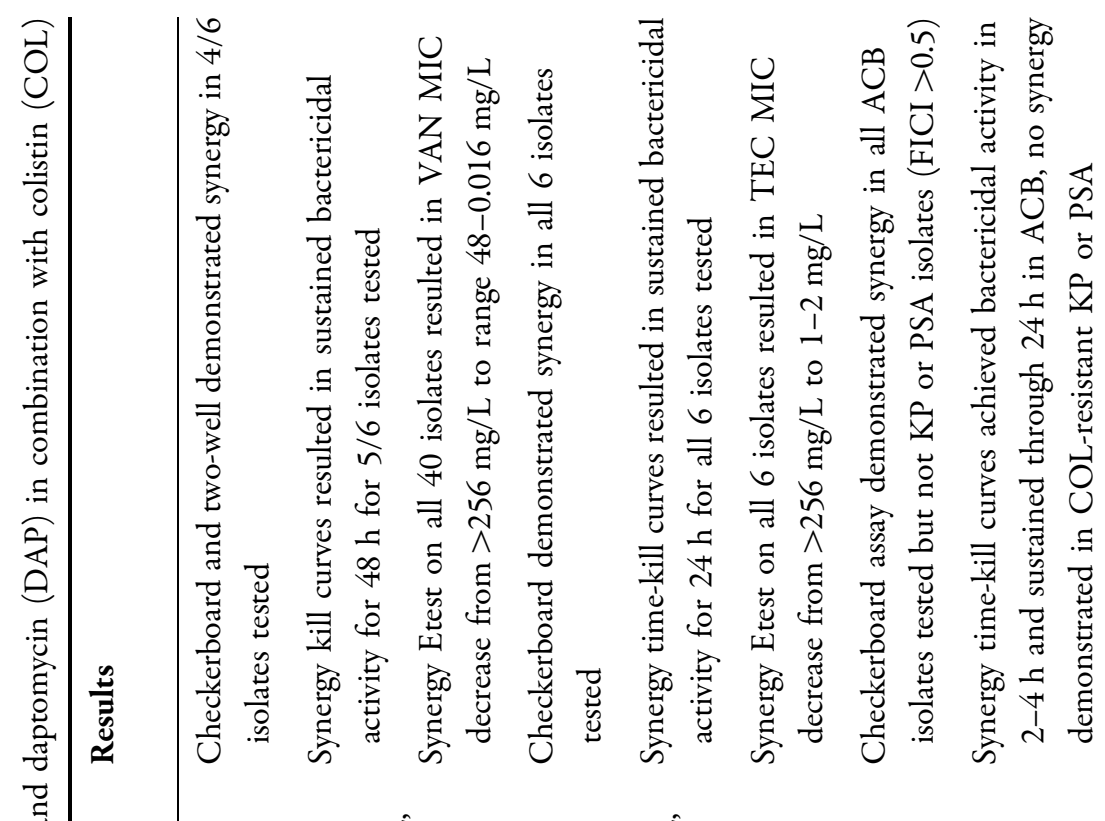

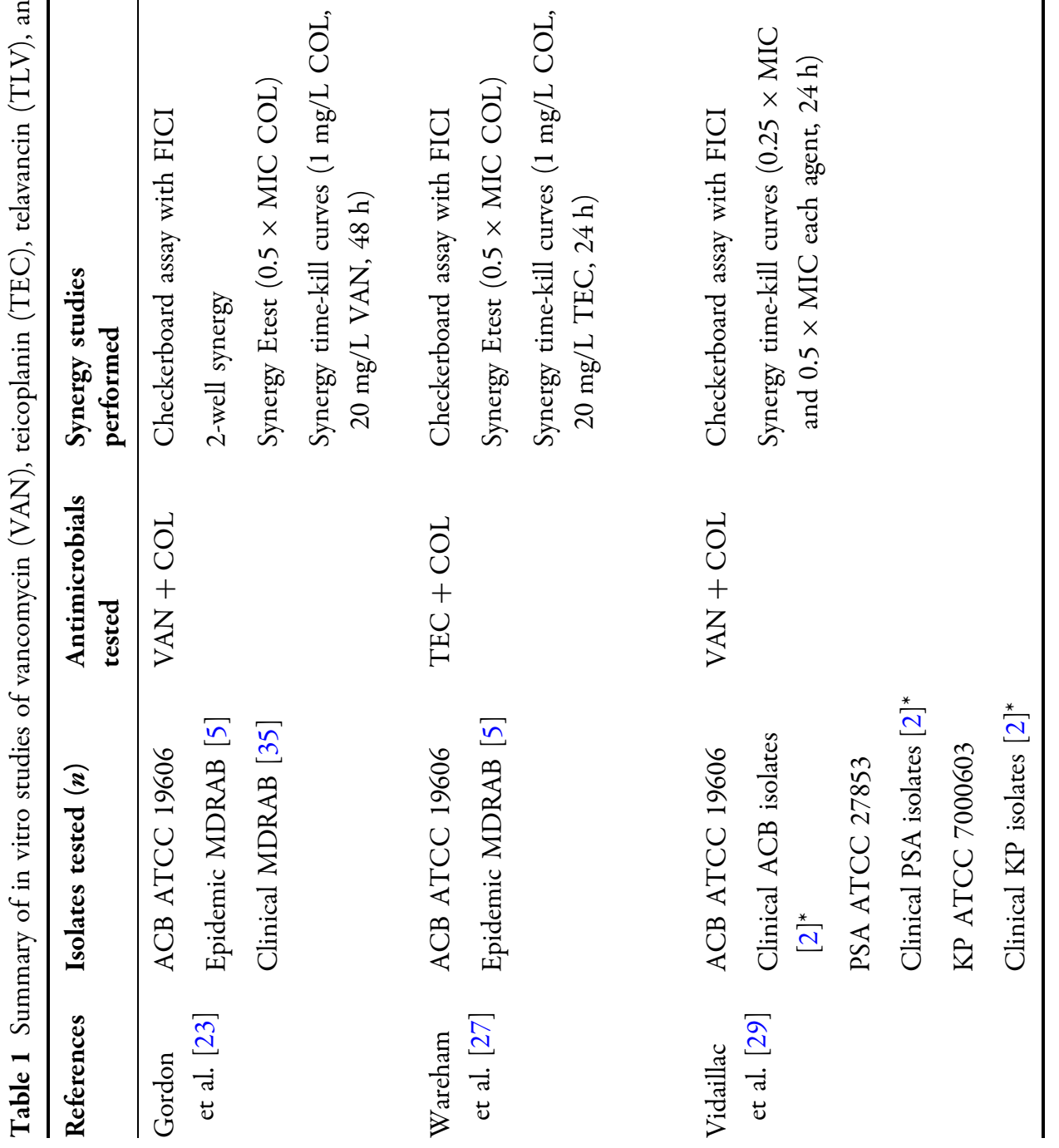




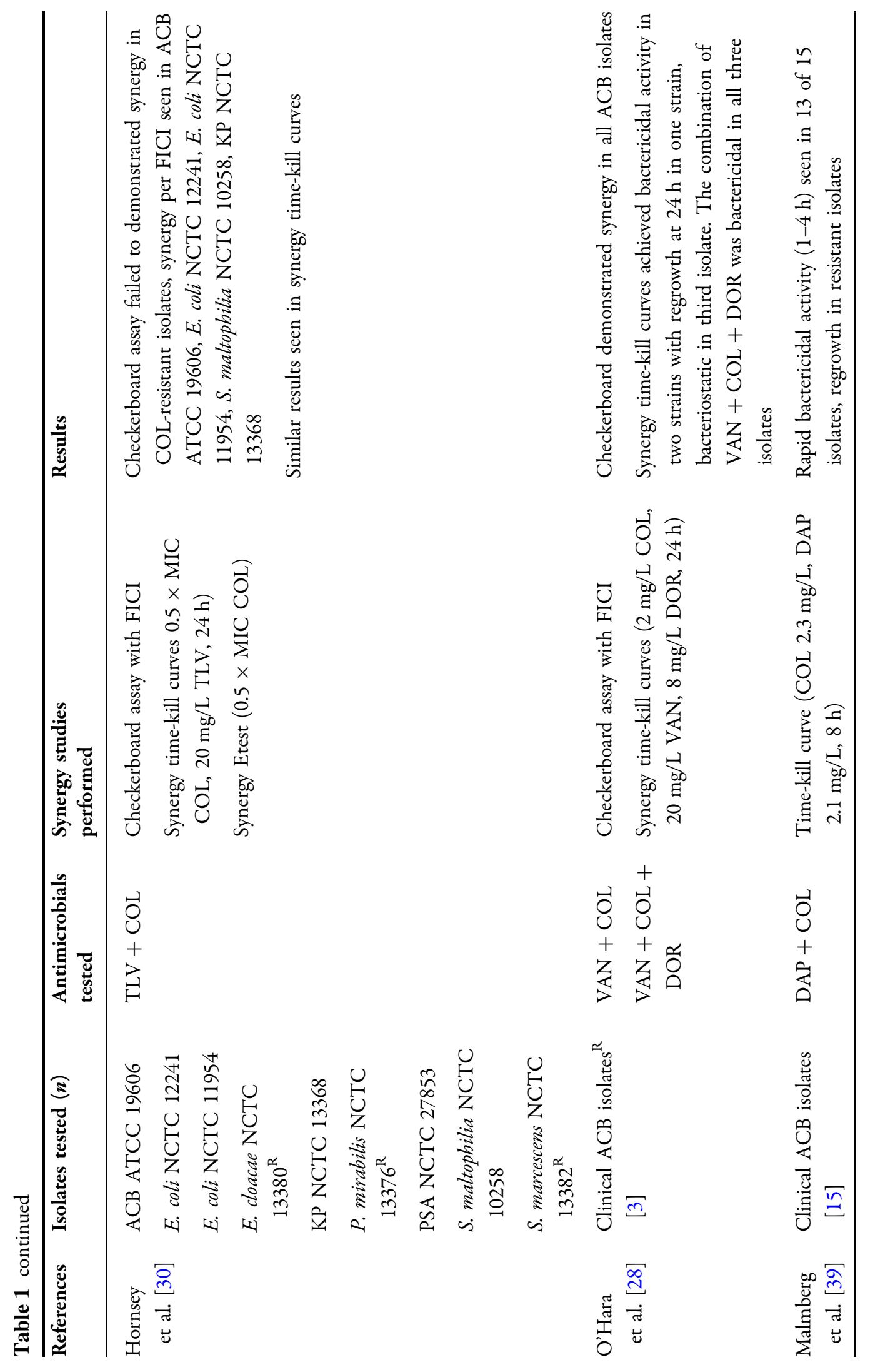




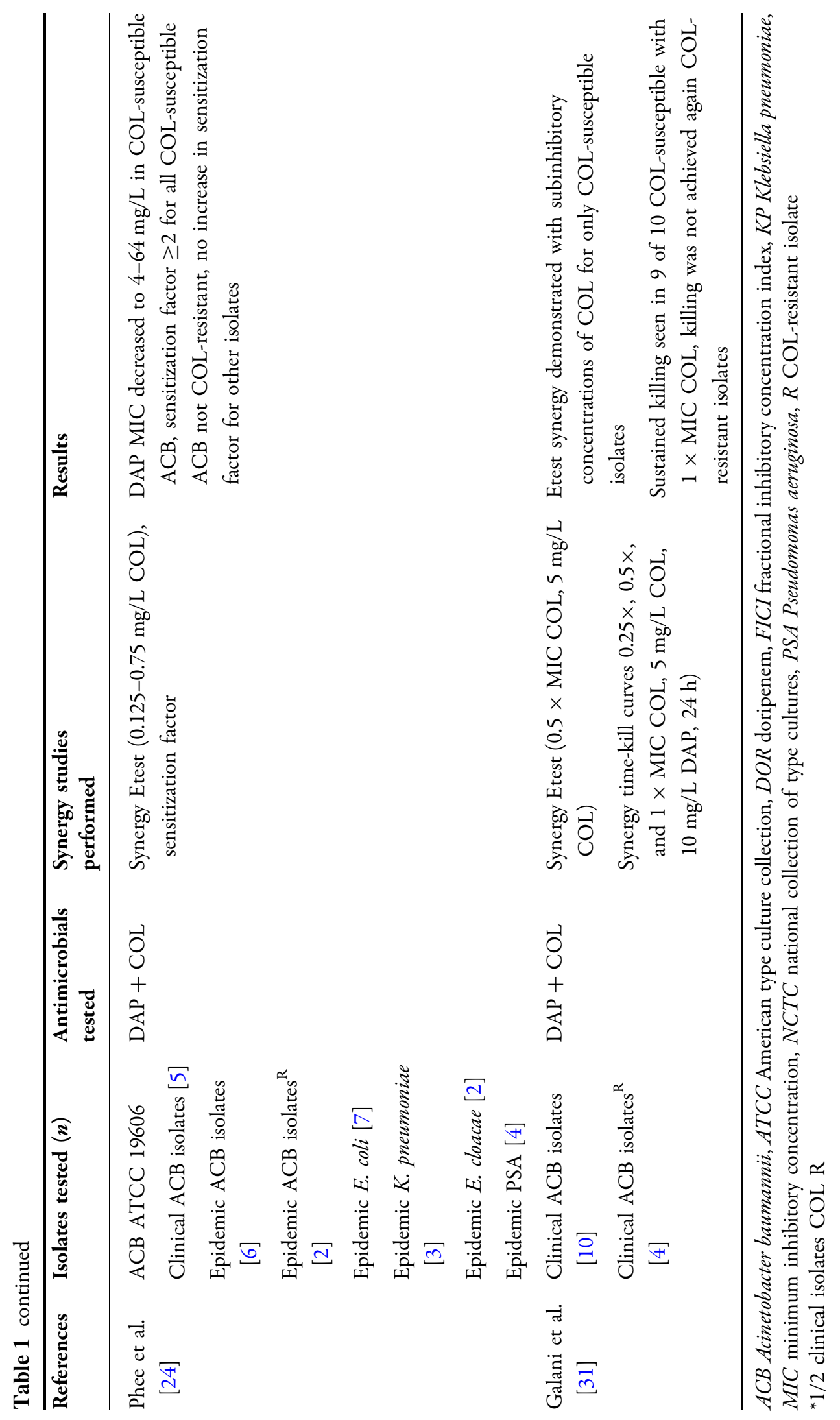



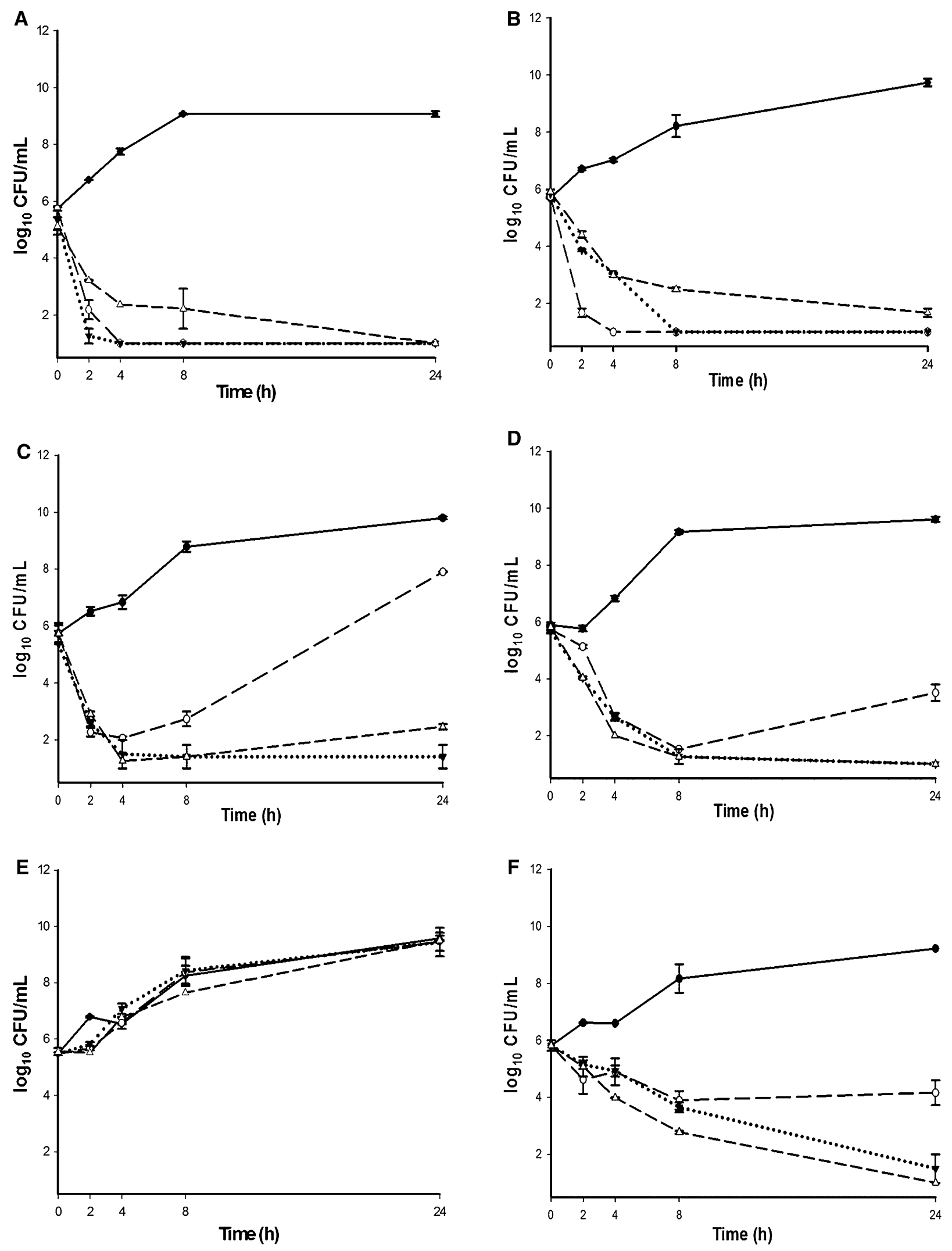
4 Fig. 1 Vancomycin and colistin in Gram-negative reference and MDR isolates. In vitro evaluation of the bactericidal activity of colistin combinations at $0.5 \times$ MIC against $A$. baumannii ATCC 19606 (a), K. pneumoniae ATCC 700603 (b), P. aeruginosa ATCC 27853 (c), $\quad \mathrm{ABm} 1 \quad[\mathrm{COL} \quad \mathrm{MIC}=8] \quad(\mathbf{d}), \quad \mathrm{KPm} 1 \quad[\mathrm{COL}$ $\mathrm{MIC}=32](\mathbf{e})$, and PAm 1 [COL $\mathrm{MIC}=8](\mathbf{f})$. Filled circle growth control, open circle colistin plus vancomycin, inverted filled triangle colistin plus trimethoprim, open triangle colistin plus trimethoprim-sulfamethoxazole. $A T C C$ American type culture collection, COL combination with colistin, $M D R$ multidrug resistant, $M I C$ minimum inhibitory concentration. Reproduced with permission from Antimicrobial Agents and Chemotherapy [29]

patients were primarily infected with carbapenem-resistant Klebsiella pneumoniae and MDR P. aeruginosa. Overall, 68 (40.9\%) of included patients received combination therapy, primarily with vancomycin. The most notable difference between patients treated with colistin alone and those treated with combination therapy was the presence of Gram-positive co-infection $\quad(41.2 \%$ versus $0.0 \%, p<0.001)$. Thirty-day mortality was not significantly different between those treated with the combination and those treated with monotherapy (33.8\% versus $29.6 \%$ ). Although infection with MDRAB was an independent predictor of mortality, through cox regression the receipt of the combination for $\geq 5$ days was shown to be protective for 30-day survival. There was no difference in renal toxicity between the patients who received glycopeptides and those that did not.

\section{Studies with Lipopeptide Antibiotics}

In addition to the glycopeptides, the lipopeptide antibiotic daptomycin and lipoglycopeptide antibiotic telavancin have been investigated in combination with colistin (Table 1). Telavancin has a dual mechanism of action wherein it acts as glycopeptide antibiotics by inhibiting transpeptidation and transglycosylation, the peptidoglycan polymerization step of cell wall synthesis, as well as altering cell membrane permeability via changing membrane potential $[35,36]$. Similar to vancomycin and teicoplanin, the combination with colistin has demonstrated significant synergy in vitro and improved survival in an in vivo invertebrate model [30, 37]. Daptomycin has a unique and not yet fully elucidated mechanism of action that causes calcium-dependent destabilization of the cell membrane as well as altering the cell wall through septal defects [38]. As is the case with glycopeptides, the presence of a LPS outer membrane in Gram-negative bacteria protects against daptomycin binding to the cellular membrane.

In a 2012 abstract, Malmberg et al. [39] initially demonstrated possible synergistic activity of daptomycin in combination with colistin against 15 clinical isolates of MDRAB. The combination of $2.3 \mathrm{mg} / \mathrm{L}$ colistin and $2.1 \mathrm{mg} / \mathrm{L}$ daptomycin was effective in 13 isolates, however, regrowth after $4 \mathrm{~h}$ was seen. Galani et al. [31] studied the combination of daptomycin and colistin against 14 MDRAB clinical isolates. Of these, four were resistant to colistin. Synergy was tested using Etests, MICs, and time-kill curves. Daptomycin Etests were placed on agar supplemented with subinhibitory concentrations $(0.5 \times$ MIC) of colistin. The timekill curves were completed with cation-adjusted Muller-Hinton broth over a 24-h period using $0.25 \times, 0.5 \times$, and $1 \times$ MIC of colistin for susceptible strains, $5 \mathrm{mg} / \mathrm{L}$ colistin for resistant strains, and $10 \mathrm{mg} / \mathrm{L}$ of daptomycin. In the Etests, subinhibitory concentrations of colistin were able to decrease the daptomycin MIC to $4-128 \mathrm{mg} / \mathrm{L}$ in the colistin-susceptible isolates but colistin concentrations upwards of $5 \mathrm{mg} / \mathrm{L}$ had no effect on daptomycin MICs for resistant isolates. A total of 30 isolate-concentration 
combinations were performed in the time-kill curves. Daptomycin and colistin demonstrated synergy against 16 of the 30 (53.3\%) combinations. Among ten colistin-susceptible isolates that were exposed to $0.5 \times$ MIC colistin bactericidal activity occurred in five (50\%), compared to zero isolates exposed without daptomycin. Nine of 10 isolates exposed to colistin at $1 \times$ MIC demonstrated bactericidal activity with no regrowth at $24 \mathrm{~h}$. It appears the possible benefits of the daptomycin-colistin combination were limited to colistinsusceptible isolates and favorable results were demonstrated at $0.5 \times \mathrm{MIC}$ and $1 \times \mathrm{MIC}$ for colistin.

Phee et al. [24] tested susceptibility to the daptomycin-colistin combination in 30 Gramnegative isolates, including Escherichia coli, $K$. pneumoniae, and ACB. Among the fourteen ACB isolates tested was ATCC strain 19606 and two colistin-resistant isolates. Synergy was tested using daptomycin Etests on agar supplemented with subinhibitory concentrations $(0.125-0.75 \mathrm{mg} / \mathrm{L})$ of colistin versus unsupplemented agar. The results for each were compared using a "sensitization factor" (ratio daptomycin MIC alone to MIC in combination with colistin) with a factor $\leq 2$ noting a lack of synergy. MICs for daptomycin on colistin-supplemented agar were compared to Clinical and Laboratory Standards Institute (CLSI) breakpoints for both $S$. aureus and enterococci spp. Daptomycin MICs for all isolates were initially $>256 \mathrm{mg} / \mathrm{L}$, a decrease in MIC (4-64 mg/L) and increase in sensitization factor (8-128) were observed in all colistinsusceptible ACB isolates, but not the two colistin-resistant or any other Gram-negatives tested. The author hypothesized that although potent synergy was demonstrated in ACB isolates the target for daptomycin may be absent in other Gram-negative bacteria.
Although most antibiotic agents possessing activity limited to Gram-positive pathogens may become active against Gram-negatives if allowed to pass the outer LPS layer, this is not true for daptomycin [40]. Daptomycin has to interact with the negatively charged phospholipids within the cell membrane. Randall et al. [40] hypothesized that the substantially lower amount of phospholipids (one-third that of $S$. aureus) in Gram-negatives such as E. coli may result in the lack of activity of daptomycin. After an in vitro study of the destabilizing effects of daptomycin against liposomes comprised both E. coli and S. aureus, the authors concluded that major differences in phospholipid composite affect daptomycin activity in Gram-negatives.

\section{CONCLUSION}

Gram-negative organisms such as ACB, in particular MDRAB, represent a serious threat to public health. Currently, fighting these infections often requires using last resort agents, such as colistin, that often have suboptimal activity when used as monotherapy and are associated with serious toxicities such as nephrotoxicity. Combination therapy with various antimicrobial agents has proven beneficial in the treatment of MDRAB; however, data are limited to retrospective studies of small sample sizes. Promising novel combinations of colistin with Gram-positive active agents such as glycopeptide or lipopeptide antibiotics have been studied primarily in vitro and in vivo. Future investigation to gain insight on the clinical impact of these combinations is required to determine the possible benefits associated with use of such combinations. In particular, elucidation of the mechanism by which these combinations seem to work best in $\mathrm{ACB}$ as 
opposed to other MDR Gram-negative pathogens and further investigation of whether higher doses of daptomycin will improve results in MDRAB [24, 40, 41]. Additionally, further description of use of these combinations in monomicrobial MDRAB infections without de-escalation to colistin monotherapy following eradication of coinfecting Gram-positive pathogens would be beneficial from a clinical perspective. It is also unclear if utilizing polymyxin B in lieu of colistin would provide similar synergistic activity against ACB or other MDR Gramnegative pathogens. Lastly, triple therapy with colistin, a lipopeptide or glycopeptide, and another agent active against MDRAB such as tigecycline or carbapenems should also be investigated in the clinical setting. With a dwindling pipeline of agents with novel mechanisms of action, finding new and unique means to employ antibiotics that are currently available is crucial to improve outcomes in these life-threatening infections.

\section{ACKNOWLEDGMENTS}

No funding or sponsorship was received for this study or publication of this article. All named authors meet the ICMJE criteria for authorship for this manuscript, take responsibility for the integrity of the work as a whole, and have given final approval for the version to be published.

Conflict of interest. Michael J. Rybak has received grant support, speaker or consulting fees from Cubist, Durata, Forest, Melinta, Sunovian and Theravance in addition to grant support from the NIH.

Kimberly C. Claeys and Anna D. Fiorvento have no potential conflicts of interest to declare.
Compliance with ethics guidelines. The analysis in this article is based on previously conducted studies, and does not involve any new studies of human or animal subjects performed by any of the authors.

Open Access. This article is distributed under the terms of the Creative Commons Attribution Noncommercial License which permits any noncommercial use, distribution, and reproduction in any medium, provided the original author(s) and the source are credited.

\section{REFERENCES}

1. Cooper MA, Shlaes D. Fix the antibiotics pipeline. Nature. 2011;472(7341):32.

2. Center for Disease Control and Prevention (CDC). Antibiotic Resistance Threats in the United States. http://www.cdc.gov/drugresistance/threat-report2013/ (2013).

3. May M. Drug development: time for teamwork. Nature. 2014;509(7498):S4-5.

4. Enfield KB, Huq NN, Gosseling MF, Low DJ, Hazen $\mathrm{KC}$, Toney DM, et al. Control of simultaneous outbreaks of carbapenemase-producing enterobacteriaceae and extensively drug-resistant Acinetobacter baumannii infection in an intensive care unit using interventions promoted in the centers for disease control and prevention 2012 carbapenemase-resistant enterobacteriaceae toolkit. Infect Control Hosp Epidemiol. 2014;35(7):810-7.

5. Farrell DJ, Sader HS, Flamm RK, Jones RN. Ceftolozane/tazobactam activity tested against Gram-negative bacterial isolates from hospitalised patients with pneumonia in US and European medical centres (2012). Int J Antimicrob Agents. 2014;43(6):533-9.

6. Flamm RK, Farrell DJ, Sader HS, Jones RN. Ceftazidime/avibactam activity tested against Gram-negative bacteria isolated from bloodstream, pneumonia, intra-abdominal and urinary tract infections in US medical centres (2012). J Antimicrob Chemother. 2014;69(6):1589-98.

7. Boucher HW, Talbot GH, Bradley JS, Edwards JE, Gilbert D, Rice LB, et al. Bad bugs, no drugs: no ESKAPE! An update from the Infectious Diseases 
Society of America. Clin Infect Dis. 2009;48(1):1-12.

8. Boucher HW, Talbot GH, Benjamin DK Jr, Bradley J, Guidos RJ, Jones RN, et al. $10 \times$ '20 Progressdevelopment of new drugs active against gramnegative bacilli: an update from the Infectious Diseases Society of America. Clin Infect Dis. 2013;56(12):1685-94.

9. Villegas MV, Hartstein AI. Acinetobacter outbreaks, 1977-2000. Infect Control Hosp Epidemiol. 2003;24(4):284-95.

10. Nutman A, Glick R, Temkin E, Hoshen M, Edgar R, Braun $\mathrm{T}$, et al. A case-control study to identify predictors of 14-day mortality following carbapenem-resistant Acinetobacter baumannii bacteremia. Clin Microbiol Infect. 2014. doi:10. 1111/1469-0691.12716.

11. Michalopoulos A, Falagas ME, Karatza DC, Alexandropoulou P, Papadakis E, Gregorakos L, et al. Epidemiologic, clinical characteristics, and risk factors for adverse outcome in multiresistant gram-negative primary bacteremia of critically ill patients. Am J Infect Control. 2011;39(5):396-400.

12. Eberle BM, Schnuriger B, Putty B, Barmparas G, Kobayashi L, Inaba $\mathrm{K}$, et al. The impact of Acinetobacter baumannii infections on outcome in trauma patients: a matched cohort study. Crit Care Med. 2010;38(11):2133-8.

13. Fournier PE, Vallenet D, Barbe V, Audic S, Ogata H, Poirel L, et al. Comparative genomics of multidrug resistance in Acinetobacter baumannii. PLoS Genet. 2006;2(1):e7.

14. Singh $\mathrm{H}$, Thangaraj $\mathrm{P}$, Chakrabarti A. Acinetobacter baumannii: a brief account of mechanisms of multidrug resistance and current and future therapeutic management. J Clin Diagn Res. 2013;7(11):2602-5.

15. Kiratisin P, Chongthaleong A, Tan TY, Lagamayo E, Roberts S, Garcia J, et al. Comparative in vitro activity of carbapenems against major Gramnegative pathogens: results of Asia-Pacific surveillance from the COMPACT II study. Int J Antimicrob Agents. 2012;39(4):311-6.

16. Chopra S, Torres-Ortiz M, Hokama L, Madrid P, Tanga M, Mortelmans K, et al. Repurposing FDAapproved drugs to combat drug-resistant Acinetobacter baumannii. J Antimicrob Chemother. 2010;65(12):2598-601.

17. Velkov T, Roberts KD, Nation RL, Thompson PE, Li J. Pharmacology of polymyxins: new insights into an 'old' class of antibiotics. Future Microbiol. 2013;8(6):711-24.
18. Dhariwal AK, Tullu MS. Colistin: re-emergence of the 'forgotten' antimicrobial agent. J Postgrad Med. 2013;59(3):208-15.

19. Paul M, Carmeli Y, Durante-Mangoni E, Mouton JW, Tacconelli E, Theuretzbacher $\mathrm{U}$, et al. Combination therapy for carbapenem-resistant Gram-negative bacteria. J Antimicrob Chemother. 2014;69(9):2305-9.

20. Sirijatuphat R, Thamlikitkul V. Colistin versus colistin plus fosfomycin for treatment of carbapenem-resistant Acinetobacter baumannii infections: a preliminary study. Antimicrob Agents Chemother. 2014;58(9):5598-601.

21. Poulikakos P, Tansarli GS, Falagas ME. Combination antibiotic treatment versus monotherapy for multidrug-resistant, extensively drug-resistant, and pandrug-resistant Acinetobacter infections: a systematic review. Eur J Clin Microbiol Infect Dis. 2014;33(10):1675-85.

22. Batirel A, Balkan II, Karabay O, Agalar C, Akalin S, Alici $\mathrm{O}$, et al. Comparison of colistin-carbapenem, colistin-sulbactam, and colistin plus other antibacterial agents for the treatment of extremely drug-resistant Acinetobacter baumannii bloodstream infections. Eur J Clin Microbiol Infect Dis. 2014;33(8):1311-22.

23. Gordon NC, Png K, Wareham DW. Potent synergy and sustained bactericidal activity of a vancomycincolistin combination versus multidrug-resistant strains of Acinetobacter baumannii. Antimicrob Agents Chemother. 2010;54(12):5316-22.

24. Phee L, Hornsey M, Wareham DW. In vitro activity of daptomycin in combination with low-dose colistin against a diverse collection of Gramnegative bacterial pathogens. Eur J Clin Microbiol Infect Dis. 2013;32(10):1291-4.

25. Rybak MJ, Rotschafer JC, Rodvold KA. Vancomycin: over 50 years later and still a work in progress. Pharmacotherapy. 2013;33(12):1253-5.

26. Koyama N, Inokoshi J, Tomoda H. Anti-infectious agents against MRSA. Molecules. 2012;18(1):204-24.

27. Wareham DW, Gordon NC, Hornsey M. In vitro activity of teicoplanin combined with colistin versus multidrug-resistant strains of Acinetobacter baumannii. J Antimicrob Chemother. 2011;66(5):1047-51.

28. O'Hara JA, Ambe LA, Casella LG, Townsend BM, Pelletier MR, Ernst RK, et al. Activities of vancomycin-containing regimens against colistinresistant Acinetobacter baumannii clinical strains. Antimicrob Agents Chemother. 2013;57(5):2103-8. 
29. Vidaillac C, Benichou L, Duval RE. In vitro synergy of colistin combinations against colistin-resistant Acinetobacter baumannii, Pseudomonas aeruginosa, and Klebsiella pneumoniae isolates. Antimicrob Agents Chemother. 2012;56(9):4856-61.

30. Hornsey M, Longshaw C, Phee L, Wareham DW. In vitro activity of telavancin in combination with colistin versus Gram-negative bacterial pathogens. Antimicrob Agents Chemother. 2012;56(6):3080-5.

31. Galani I, Orlandou K, Moraitou H, Petrikkos G, Souli M. Colistin/daptomycin: an unconventional antimicrobial combination synergistic in vitro against multidrug-resistant Acinetobacter baumannii. Int J Antimicrob Agents. 2014;43(4):370-4.

32. Hornsey M, Wareham DW. In vivo efficacy of glycopeptide-colistin combination therapies in a Galleria mellonella model of Acinetobacter baumannii infection. Antimicrob Agents Chemother. 2011;55(7):3534-7.

33. Garnacho-Montero J, Amaya-Villar R, GutierrezPizarraya A, Espejo-Gutierrez de Tena E, ArteroGonzalez ML, Corcia-Palomo Y, et al. Clinical efficacy and safety of the combination of colistin plus vancomycin for the treatment of severe infections caused by carbapenem-resistant Acinetobacter baumannii. Chemotherapy. 2013;59(3):225-31.

34. Petrosillo N, Giannella M, Antonelli M, Antonini M, Barsic B, Belancic L, et al. Clinical experience of colistin-glycopeptide combination in critically ill patients infected with Gram-negative bacteria. Antimicrob Agents Chemother. 2014;58(2):851-8.
35. Zhanel GG, Calic D, Schweizer F, Zelenitsky S, Adam H, Lagace-Wiens PR, et al. New lipoglycopeptides: a comparative review of dalbavancin, oritavancin and telavancin. Drugs. 2010;70(7):859-86.

36. Saravolatz LD, Stein GE, Johnson LB. Telavancin: a novel lipoglycopeptide. Clin Infect Dis. 2009;49(12):1908-14.

37. Hornsey M, Phee L, Longshaw C, Wareham DW. In vivo efficacy of telavancin/colistin combination therapy in a Galleria mellonella model of Acinetobacter baumannii infection. Int J Antimicrob Agents. 2013;41(3):285-7.

38. Pogliano J, Pogliano N, Silverman JA. Daptomycinmediated reorganization of membrane architecture causes mislocalization of essential cell division proteins. J Bacteriol. 2012;194(17):4494-504.

39. Malmberg CAL, Bertiolsson-Forsberg P, Lagerback P. Colistin in combination with daptomycin enhances the effect against $A$. baumannii. London: European Congress of Clinical Microbiology and Infectious Diseases (ECCMID); 2012.

40. Randall CP, Mariner KR, Chopra I, O'Neill AJ. The target of daptomycin is absent from Escherichia coli and other gram-negative pathogens. Antimicrob Agents Chemother. 2013;57(1):637-9.

41. Tangden T, Hickman RA, Forsberg P, Lagerback P, Giske CG, Cars O. Evaluation of double- and tripleantibiotic combinations for VIM- and NDMproducing Klebsiella pneumoniae by in vitro timekill experiments. Antimicrob Agents Chemother. 2014;58(3):1757-62. 